\title{
Doppler Global Velocimetry \\ The Next Generation?
}

\author{
by \\ James F. Meyers \\ NASA - Langley Research Center \\ Hampton, Virginia 23665
}

\begin{abstract}
A new measurement technique is being developed by NASA to measure off-surface flow fields. This method, Doppler global velocimetry, will allow quantification of complex three-dimensional flow fields at video camera rates. The entire flow field structure within a selected plane is measured simultaneously rather than by scanned, point-by-point measurements using conventional laser velocimetry. To assess the capability of this new technique, velocity measurements of the vortical flow field above a thin 75-degree delta wing were made in the NASA Langley Basic Aerodynamics Research Tunnel. The operation of the system is described along with the techniques developed to process the output images. The results are then compared with similar measurements obtained using a three component laser velocimeter.
\end{abstract}

\section{Nomenclature}

ALF Absorption line filter, Iodine vapor

c Speed of light, $\mathrm{m} / \mathrm{sec}$

$\hat{1} \quad$ Laser beam propagation direction

ô Collected scattered light direction

$\mathrm{V}$ Velocity of a particle passing through the laser beam, m/sec

$X \quad$ Cross tunnel coordinate, $\mathrm{m}$

$Y \quad$ Vertical coordinate, $\mathrm{m}$

Z Streamwise coordinate, $\mathrm{m}$

$\Delta v \quad$ Doppler shift frequency, $\mathrm{Hz}$ 
$\theta \quad$ Angle between the laser propagation direction and the collected scattered light, deg

\section{Introduction}

Fringe-type laser velocimetry has demonstrated over the last two decades that nonintrusive flow diagnostics can yield measurements of the quantity and quality necessary to describe the flow velocity structure in fluid mechanic investigations. The technique has provided insights into the three component mean flow characteristics, turbulence intensities, Reynolds stresses and turbulence power spectra. While no other instrumentation technique, even intrusive methods, have yielded such a wealth of information describing the structure of basic fluid mechanics, the present demands of state-of-theart computational fluid dynamics have exceeded the capabilities of fringe-type laser velocimetry. Being a point measurement, large amounts of time are required to map an entire flow field. This characteristic requires the flow to be stationary over the possibly many hours necessary to make these measurements.

Since it is difficult to maintain flow stationarity for these long times, researchers began to look for techniques that could measure the flow parameters in less time. The first approach was to rapidly scan the sample volume of a classic fringe-type laser velocimeter, reference 1. This technique was successful in time varying flow fields, but required the same amount of measurement time as the point-by-point approach if accurate measurements were desired. The development of particle image velocimetry was the first truly global velocity measurement technique, reference 2. It uses a double pulse laser to illuminate a measurement plane within the flow. Scattered light from the particles present within the plane is collected and imaged on photographic film. The double exposures recorded on film are then interrogated to determine the distance traveled by each recorded particle during the time between pulses. Thus the velocity field of the flow passing through the light sheet can be obtained instantaneously. While a major advance in the state-of-the-art, this technique has its drawbacks: limited viewing area (typically 4 x 5 -inches), limited to two velocity component measurements, directional ambiguity, and long data processing times. Although these drawbacks may be reduced, or in some cases even eliminated with further research, a new technique - Doppler Global Velocimetry - is proposed as a new global measurement system which may provide even more information while reducing instrument complexity - A new generation in measurement technology? 


\section{Basic Principles}

The fundamental physics governing the Doppler global velocimeter, DGV, is based on the shift in optical frequency of scattered light from objects passing through a laser beam. This principle was first exploited by Yeh and Cummins in 1964, reference 3, to develop the reference beam, laser Doppler velocimeter, LDV. As depicted in figure 1, scattered light collected by a detector located along the direction ô, from particles passing through a laser beam propagating in direction $\hat{1}$, is Doppler shifted based on a velocity in the direction (ô $-\hat{1})$. This relationship is expressed by:

$$
\Delta v=\frac{v_{o}(\hat{o}-\hat{1}) \bullet V}{c}
$$

where $\Delta v$ is the Doppler shifted frequency, $v$ is the laser frequency, $\mathbf{V}$ is the particle velocity, and $c$ is the speed of light. The angular dependence of the Doppler shift magnitude on system geometry, as indicated by the dot product in equation (1), presents the possibility of three component velocity measurements by using various laser propagation directions and/or locations for the receiver optical system.

Although equation (1) indicates that the Doppler shift principle provides the necessary measurable parameter, the instrumentation task is to develop a method to measure that parameter. Yeh and Cummins mixed a portion of the scattered light with unshifted laser light on a photocathode surface to obtain heterodyne detection, thus isolating the Doppler frequency. Whereas Self, reference 4, used a Fabry-Perot interferometer to measure the shifted light frequency directly. These techniques have characteristically poor signal-to-noise ratios, and complicated, alignment sensitive, optical systems. A more robust system using a Michelson interferometer was constructed by Smeets, reference 5. As the fringes produced by the interferometer pass over a spatially filtered photomultiplier, an electronic signal containing the Doppler frequency is output. While this technique increases the signal-to-noise ratio, it still requires a complicated, alignment sensitive, optical system. Komine, et al, reference 6 , realized that the optical transfer function of an absorption line in Iodine vapor could be used as an optical frequency discriminator. An Argon ion laser operating in single-line mode at $514.5 \mathrm{~nm}$ is tuned by tilting the intercavity etalon to an optical frequency corresponding to a point midway along the edge of an Iodine absorption line, figure 2. Collected scattered light from a stationary object or cloud of particles will be attenuated by 50 percent as it passes through the Iodine vapor. If the object or particle cloud is moving, the attenuation through the vapor will increase (or decrease, depending on the direction of movement) by 
an amount proportional to the Doppler shift. This simple idea has resulted in a velocity measuring technique that has the potential for good signal-to-noise ratios, permitting accurate measurements, while keeping the optical system simple. In practice, particle size distribution and number density, and the laser beam intensity profile are other factors which influence the intensity of the scattered light. Therefore, a second detector is used to measure a portion of the collected scattered light, thus serving as a reference of absolute scattered light intensity. Normalization of the signal detector output, by the reference detector output, results in a signal whose intensity is directly related to the Doppler shift of the scattered light.

While the discussion has been limited to point measurement techniques, the Michelson interferometer and the Iodine cell methods can be expanded to global measurement systems. The expansion consists of fanning the laser beam into a light sheet and replacing the detectors with CCD video cameras. The Michelson interferometer technique, as implemented by Seiler and Srulijes, reference 7, produces images with fringe patterns indicating lines of constant phase shift outlining contours of constant velocity. Their work in supersonic flow indicates the potential of the interferometer to produce Doppler pictures in high-speed, short run time facilities. The Iodine cell technique or Doppler global velocimetry, as implemented by Meyers and Komine, reference 8, produces images whose intensity at any pixel is a measure of the average velocity of the flow passing through the light sheet viewed by that pixel. Although these two techniques have many similarities, the Doppler global velocimeter is the method of choice since it has a simpler optical system and its measurement images are easier to interpret.

\section{The Doppler Global Velocimeter}

A research program is underway at NASA to implement the principles of Doppler global velocimetry in a prototype instrumentation system that can be applied to wind tunnel and flight applications. This system was configured as illustrated in figure 3 for the proof-of-concept wind tunnel investigation in the NASA - Langley Research Center, Basic Aerodynamic Research Tunnel. Three component measurements were obtained by routing the input laser beam through each side window and the ceiling of the test section in sequence. This placed the fixed receiver optical system, shown in figure 3 , in forward scatter, back scatter, and side scatter modes respectively. A portion of the scattered light was collected by a $35 \mathrm{~mm}$ camera lens with a focal length variable from $35 \mathrm{~mm}$ to $105 \mathrm{~mm}$. A fast, spherical transfer lens was placed behind the collecting lens to collimate the light, and pass it to a beam splitter. The 
beam splitter reflects a portion of the light along a path to the reference camera, while transmitting the remaining light along a path through the Iodine vapor cell to the signal camera.

The output images from the two CCD video cameras were acquired by a specially designed digital image processor. The processor provided the necessary synchronization signals to the two cameras insuring temporal coincidence of the integration of scattered light from the particles passing through the light sheet. The synchronized images were simultaneously recorded by parallel frame grabbers using eightbit analog-to-digital converters. The resulting digitized signal image was normalized by the digitized reference image using a table look-up method, reference 9 . The original signal and reference images along with the normalized signal image were passed to the data acquisition microcomputer for final storage. Real-time signal processing was performed using analog divider circuitry in a separate signal processing system, reference 9. Pseudo colors were added to the output video signal from the analog processor using a standard video frame grabber placed in the data acquisition microcomputer. The video signal was then recorded on an optical video disk.

\section{Signal Processing - Revisited}

On the surface, the signal processing scheme outlined in the previous section appears to be correct according to the basic principles describing the operation of the Doppler global velocimeter. However, the experiences during wind tunnel testing and the analysis of the acquired data emphasized the faults within the prototype instrumentation system and the approach used to process the signal images. The basic problem was the inability to obtain an exact overlay of the signal and reference images. Factors contributing to this inability include mismatched depths of field, and optical distortions caused by lens aberrations, imperfections in the beamsplitters and mirrors, and the Iodine vapor cell. The nonuniformity of the pixel distribution and element orientation in the cameras were other factors. But the primary factor was the inability to optically align the system to the accuracy necessary to insure that corresponding pixels in each camera view the exact same spatial volume within the light sheet. Position and parallelism of the two images were fairly straight forward to obtain, but the magnification and focus proved to be very difficult. Although these obstacles may not be insurmountable, image processing techniques were investigated as a possible mechanism to fine tune the alignment.

Since the normalized images acquired on-line were obtained using misaligned optics, they were discarded. The stored images obtained 
from the signal and reference cameras needed to be reprocessed to obtain new normalized images that have been corrected for the optical aberrations and system misalignment. The signal processor used online did not have the capability to perform image processing tasks. Thus a commercially available image processing system was used to determine the approach necessary to correct the images, then perform the normalization.

Fortunately, after every align ment of the optical system, several images were recorded of a calibration grid placed in the light sheet plane. Differences between the recorded images and the original grid were due to distortions along the optical path from the grid to the acquisition camera, and from keystone distortion caused by viewing perspective. If the images can be warped to align the recorded grid to the original grid, corresponding pixels in each camera would be assured to have viewed the same spatial volume. The image processing system has the capability to warp an image with specified fiducial points to a reference grid. Unfortunately the technique used was designed to improve a visual perception of the subject image and performed the warping based on a least squares fitting of the fiducial points to the reference grid. Since the fiducial points set by the viewed grid in the light sheet plane will be in different location s for each camera, the least square fits will be different and pixel alignment can not be obtained. Therefore custom software was written to insure the placement of the fiducial points to the reference grid, guaranteeing proper pixel alignment.

The method implemented in software was based on determining the set of linear equations needed to move viewed grid points on a calibration card from their imaged positions to their ideal positions. The calibration card consisted of square grid points, $2.5 \mathrm{~mm}$ on a side arranged in a square grid with $12.5 \mathrm{~mm}$ center-to-center separation. The determination of black grid points on a white card can normally by accomplished using amplitude threshold detection. Unfortunately, aberrations in the optical system caused the intensity to vary across the image, prohibiting the use of amplitude threshold detection. However, edge detection filtering, such as a Sobel filter, would outline the grid points regards of amplitude since it only depends on the spatial rate of change of amplitude. Thus a mask based on a thresholded Sobel filtered image was used to isolate the grid points. The resulting image was interrogated to find each grid point by triggering on non zero values. Once found, the software surveyed the adjacent pixel values to determine the perimeter of the grid point. This process continued until closure was obtained with the starting location. The perimeter locations were then enclosed by a box which served as an evaluation area. The values of each row contained within the box were collapsed to a histogram which was then statistically an alyzed to find the horizontal 
center of mass. The columns were then collapsed in the same manner and the vertical center of mass determined. This subpixel center of mass location was stored in an array as the grid point to be warped.

Once all grid points have been located, the generation of the bilinear warping coefficients began by determining the offset required to move the upper left grid point horizontally, aligning it with the first vertical grid line. This offset was applied to the entire top row. The distance from the upper left grid point to the adjacent grid point to the right was determined. It was then divided into the grid spacing to obtain the multiplying factor necessary to align the second point with the second vertical grid line. The grid points on the top row were brought closer to alignment by multiplying their respective distances from the first grid point by this first warping coefficient. The distance from the now aligned second point and the third was determined and used to obtain the next warping coefficient. The process continued for the remaining grid points on the top row, completing its alignment. This procedure was used to complete the horizontal alignment of the grid by applying it to the other rows. Usage of the technique in the vertical direction brought the grid points into coincidence with the reference grid, resulting in the determination of the full set of warping coefficients.

Instead of operating on the data image to dewarp it, the chosen technique was to select each pixel from the reference grid and use the warping coefficients to determine where that pixel would map in to the data image. The amplitude of the mapped pixel position was determined by linear interpolation among the surrounding pixel values in the data image. This process had the advantage of placing subpixel positions in the midst of known values, versus moving the data image pixels to subpixel positions within the reference grid without surrounding neighbors.

The remaining adjustment to the data was the removal of sensitivity variations among the pixels in each CCD camera. Calibration of the cameras determined that their overall transfer functions were linear. This characteristic allowed the use of flat field illumination at two intensities to determine the slope and intercept of the transfer function for each pixel. The slope and intercept arrays were used to remove the sensitivity variations of the respective camera from the data images prior to dewarping.

\section{Laboratory Experimentation}

The ability of Doppler global velocimetry to make global measurements was tested in the laboratory by attempting to measure the velocity 
distribution of a rotating wheel. The DGV transmitting optics were configured to produce a column of light incident on the wheel, angled 22 degrees to the wheel surface. The receiving optical system, in standard configuration, was placed in the horizontal plane, 54 degrees to the wheel surface. This arrangement will measure the velocity along the direction 38 degrees to the wheel surface in the horizontal plane, figure 4. This should produce measurements of maximum velocity at the top of the wheel, which then decrease in a linear manner toward a maximum velocity in the opposite direction at the bottom of the wheel. The resulting measurements are shown in figure 5 with gray scale mapping of the velocity bands. Now that the DGV has been demonstrated, its ability to measure flow fields would be tested in the NASA - Langley Basic Aerodynamics Research Tunnel.

\section{Flow Field Investigation Above a 75-degree Delta Wing}

The potential of the Doppler global velocimeter for flow field diagn ostics was investigated by attempting to measure the vortical flow field above a 75-degree delta wing in the Basic Aerodynamics Research Tunnel, BART. The facility, reference 10 , was an open return tunnel with a test section $0.71 \mathrm{~m}$ high, $1.02 \mathrm{~m}$ wide and $3.07 \mathrm{~m}$ long. A maximum velocity of $67 \mathrm{~m} / \mathrm{sec}$ could be obtained in the test section with a test Reynolds number per meter of 0.43 million. The airflow entering the test section was conditioned by a honeycomb structure, four antiturbulence screens, and an 11:1 contraction ratio. The freestream turbulence intensity was less than 0.08 percent for all flow conditions. The propylene glycol vaporization/condensation generator developed for vapor screen flow visualization was used as the source of particles for the experiment. The particles were injected upstream of the honeycomb structure and have a size distribution which peaks at $0.7 \mu \mathrm{m}$ with a skewed distribution to a maximum of $10 \mu \mathrm{m}$, reference 11 .

The stainless steel 75 -degree delta wing, $0.57 \mathrm{~m}$ in length, with sharp leading edges was placed in the tunnel at an angle of attack of 20.5 degrees. The tunnel dynamic pressure was set to $402 \mathrm{~N} / \mathrm{m}^{2}$ which yields a freestream velocity of $40 \mathrm{~m} / \mathrm{sec}$. An Argon ion laser operating in $\mathrm{TEM}_{\text {oo }}$ mode with an etalon to maintain single longitudinal mode at $514.5 \mathrm{~nm}$ was used as the light source. The output beam was directed to one of three cylindrical lenses to form a light sheet placed perpendicular to the tunnel centerline at the 70 percent chord location on the model. Each of the three cylindrical lenses were placed along an optical path to bring the light sheet into the test section from each side and the top respectively, resulting in optical configurations that would measure three velocity components. The receiver optical system was located 
53 degrees from the streamwise (tunnel centerline) direction in the horizontal plane, figure 3. A photograph of the receiver optical system viewing particles in the vortical flow passing through the light sheet above the delta wing is shown in figure 6.

The outputs from the reference and signal cameras were processed by both an analog normalization circuit and a digital dual frame grabber, reference 9 . The analog processor consists of an analog divider circuit that normalizes the signal camera output by the reference camera signal in real time. The resulting signal was converted to a standard RS-170 video signal that was stored, along with the two camera signals, on a 450-line video disk recorder. The digital dual frame grabber simultaneously acquires a video frame from both cameras. The amplitudes of the measured light intensities for a given pixel in each frame were used to address a lookup table cell containing the precomputed normalization value for those amplitudes. The resulting normalized image was passed, along with the original frames, to a microcomputer for further processing and storage. Off line, the camera data was reprocessed to remove pixel sensitivity variations, optical distortion influences, and alignment inaccuracies using the method described in the previous section.

The experiment was patterned after a previous investigation, reference 11, which used a three component, fringe-type laser velocimeter, $L V$, to measure the vortical flow field above a delta wing in BART. The results obtained by the LV, figure 7 , will be used as the standard for comparison with the present DGV measurements. The gray scale represents contours of streamwise velocity and the arrows represent the velocity vector of the circular flow within the plane perpendicular to the streamwise direction. Note that the streamwise velocity accelerated to twice the freestream value at the vortex core. The circular flow was compressed by the wing and accelerated to 1.5 times the freestream value as the flow expanded outward below the core. Of the three measurement directions established by the DGV geometry, the direction that best illustrated the circular flow characteristics of the vortex was the nearly cross flow component, 71.5 degrees from the streamwise direction in the horizontal plane, figure 8. Resolving the three component LV data to obtain the velocity field along this direction yielded the contour map shown in figure 9 . The average of 30 frames of DGV data acquired by the digital dual frame grabber of the flow field along this component is shown in figure 10 . The characteristic velocity pattern for a vortex flow in the cross flow direction is clearly seen in the LV data and the left vortex in the DGV data. However, the right vortex in the DGV data more closely matches the velocity mapping obtained from the rotating wheel, figure 5 . While the right vortex has not burst, as indicated by the other component 
measurements, it may represent the transition to a burst condition since increasing the angle-of-attack of the model results in the right vortex bursting first. This asymmetric bursting has been traced to a yaw of 0.2 degrees of the model caused by the mounting hardware.

The next component, illustrated in figure 11, is the vertical component since it is the only one of the three measured with vertical sensitivity. Resolving the LV data along this direction results in the velocity map shown in figure 12. Again 30 frames of DGV data were averaged to yield the velocity mapping shown in figure 13 . The third and streamwise component is illustrated in figure 14. The resolved LV data and the averaged DGV data are shown in figures 15 and 16, respectively.

Although the present experiment was designed to match the LV investigation as closely as possible, differences still existed. An assessment of the data indicates good agreement in the rotating components but questionable agreement in the streamwise direction. The nonoverlapping vortex centers between the LV and DGV data were traced following the test to a 0.5 degree roll of the model during the LV tests. The question whether the remaining differences can be traced to the comparison of the eight hours of data acquisition required by the LV, to effectively one second of acquisition using an experimental instrumentation system several years apart, still exists. Or, are these differences actually aerodynamic in nature? Are vortical flows, which appear to be extremely stable, instead very sensitive to the incoming flow and the relation of the model to it? The irony in these questions is that the Doppler global velocimeter may be the only measurement technique that can provide their answers.

\section{Summary}

A new measurement technique, Doppler global velocimetry, has been described, along with results from an experimental investigation to assess the potential of this technique for flow field diagnostics. Procedures to obtain the precise alignment of the optical system have been described, including the use of image processing to remove deficiencies in the physical hardware. The results of these tests, when compared to conventional laser velocimeter measurements, indicate that this robust technique is capable of describing the entire threecomponent velocity flow field simultaneously within a measurement plane in real time. Further investigations are being conducted to expand the measurement capability of the technique in velocity range, viewing plane size, real-time signal processing, and error definition and analysis. 


\section{References}

1. Gartrell, L. R.; and Jordan, F. J.: Demonstration of a Rapid-Scan Two-Dimensional Laser Velocimeter in the Langley Vortex Research Facility for Research in Aerial Application, NASA TM74081, August, 1977.

2. Adrian, R. J.: Scattering Particle Scharacteristics and Their Effect on Pulsed Laser Measurements of Fluid Flow: Speckle Velocimetry vs. Particle Image Velocimetry, Applied Optics, 23, pp. 1690-1691, 1984.

3. Yeh, Y.; and Cummins, H. Z.: Localized Fluid Flow Measurements with a He-Ne Laser Spectrometer, Applied Physics Letters, vol. 4, no. 10, pp. 176-178, May 1964.

4. Self, S. A.: Laser Doppler Anemometer for Boundary Layer Measurements in High Velocity, High Temperature MHD Channel Flows, Proceedings of the Second International Workshop on Laser Velocimetry, Purdue University, vol. II, pp. 44-67, March 2729, 1974 .

5. Smeets, G.: Laser Doppler Velocimetry with a Michelson Spectrometer, Laser Anemometry in Fluid Mechanics, Selected Papers from the First International Symposium on Applications of Laser-Doppler Anemometry to Fluid Mechanics, editors: R. J. Adrian, D. F. G. Durao, F. Durst, H. Mishina and J. H. Whitelaw, Ladoan-Instituto Superior Tecnico, pp. 355-370, 1984

6. Komine, H.; Brosnan, S. J.; Litton, A. B.; and Stappaerts, E. A.: Real Time, Doppler Global Velocimetry, AIAA 29th Aerospace Sciences Meeting, Reno, NV, paper no. AIAA-91-0337, January 710,1991 .

7. Seiler, F.; and Srulijes, J.: Doppler-Pictures of Velocity Fields, An Application to Fluid Mechanics, Proceedings of the Third International Symposium on Applications of Laser Anemometry to Fluid Mechanics, Lisbon, Portugal, paper 19.1, July 7-9, 1986.

8. Meyers, J. F.; and Komine, H.: Doppler Global Velocimetry - A New Way to Look at Velocity, ASME 4th International Conference on Laser Anemometry, Advances and Applications, Cleveland, OH, August 5-9, 1991.

9. Meyers, J. F.; Lee, J. W.; and Cavone, A. A.: Signal Processing Schemes for Doppler Global Velocimetry, IEEE - 14th 
International Congress on Instrumentation in Aerospace Simulation Facilities, Rockville, MD, October 27-31, 1991.

10. Sellers, W. L., III; and Kjelgaard, S. O.: The Basic Aerodynamics Research Tunnel - A Facility Dedicated to Code Validation, AIAA 15th Aerodynamic Testing Conference, San Diego, CA, paper no. AIAA-88-1997, May 18-20, 1988.

11. Meyers, J. F.: A Three Dimensional View of Velocity Using Lasers, 10th International Invitational Symposium on Unification of Finite Element Methods in Theory and Test, Worcester, MA, July 18-19, 1991.

12. Meyers, J. F.; and Hepner, T. E.: Measurement of Leading Edge Vortices from a Delta Wing Using a Three Component Laser Velocimeter, AIAA 15th Aerodynamic Testing Conference, San Diego, CA, paper no. AIAA-88-2024, May 18-20, 1991. 
Laser Propagation Direction, î

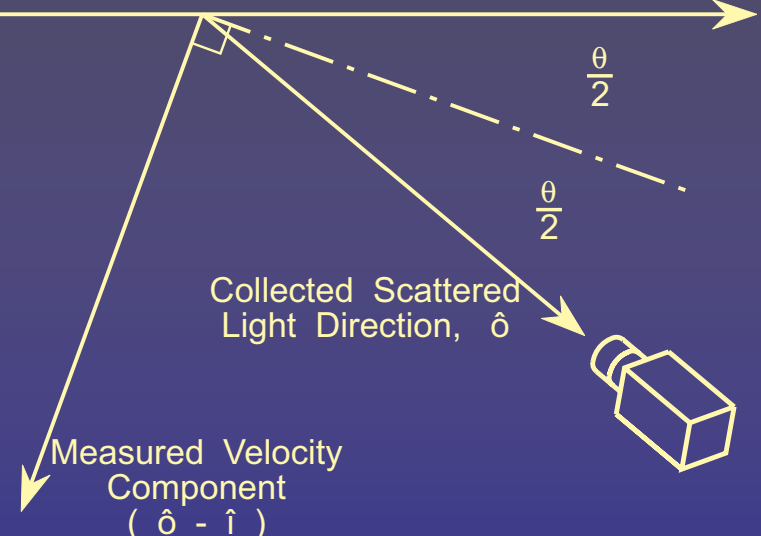

Figure 1.- Diagram depicting the velocity measurement direction based on the orientation of the laser propagation direction and the detector location.

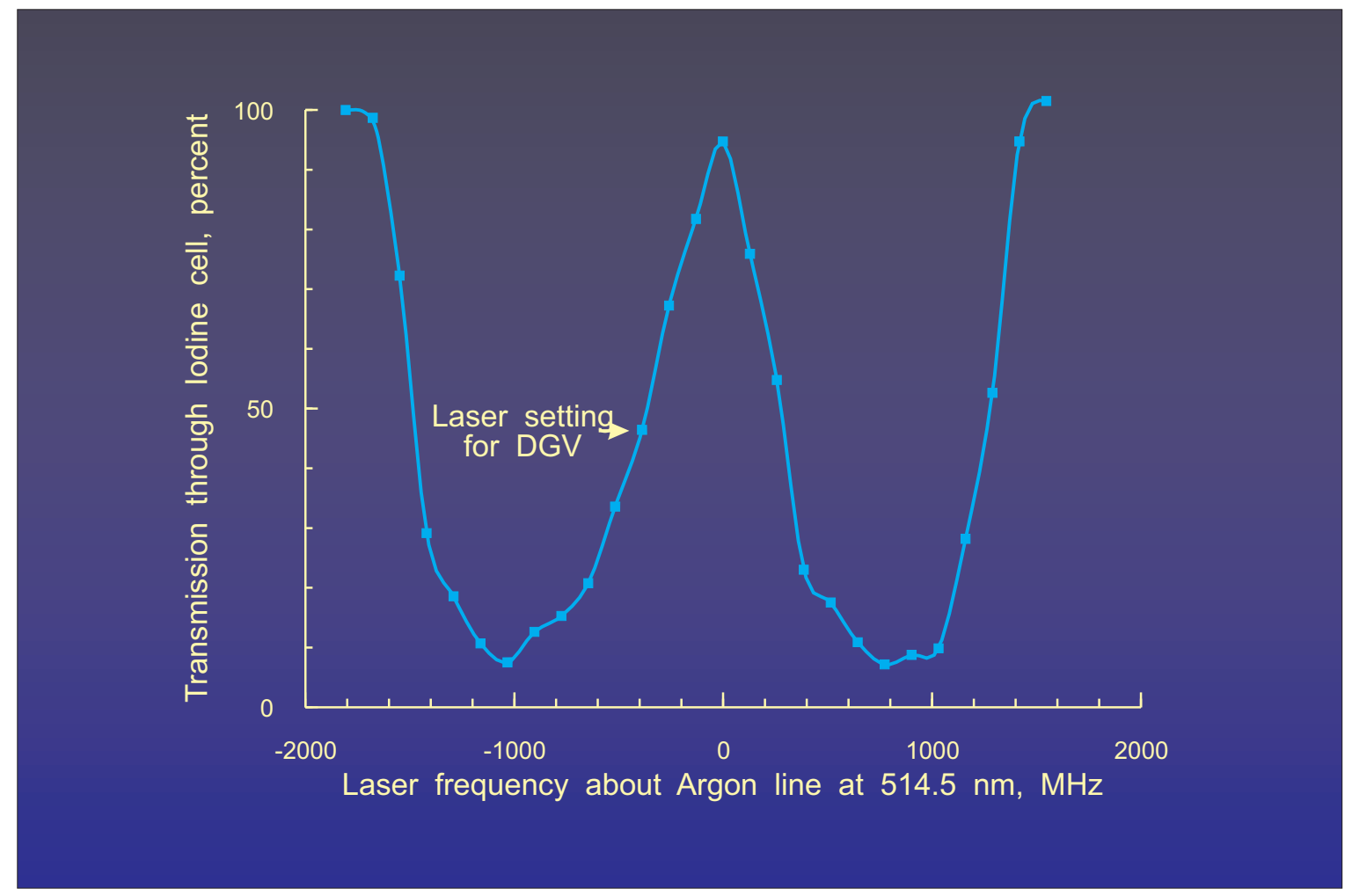

Figure 2.- Transfer function of the Iodine absorption line filter, ALF. 


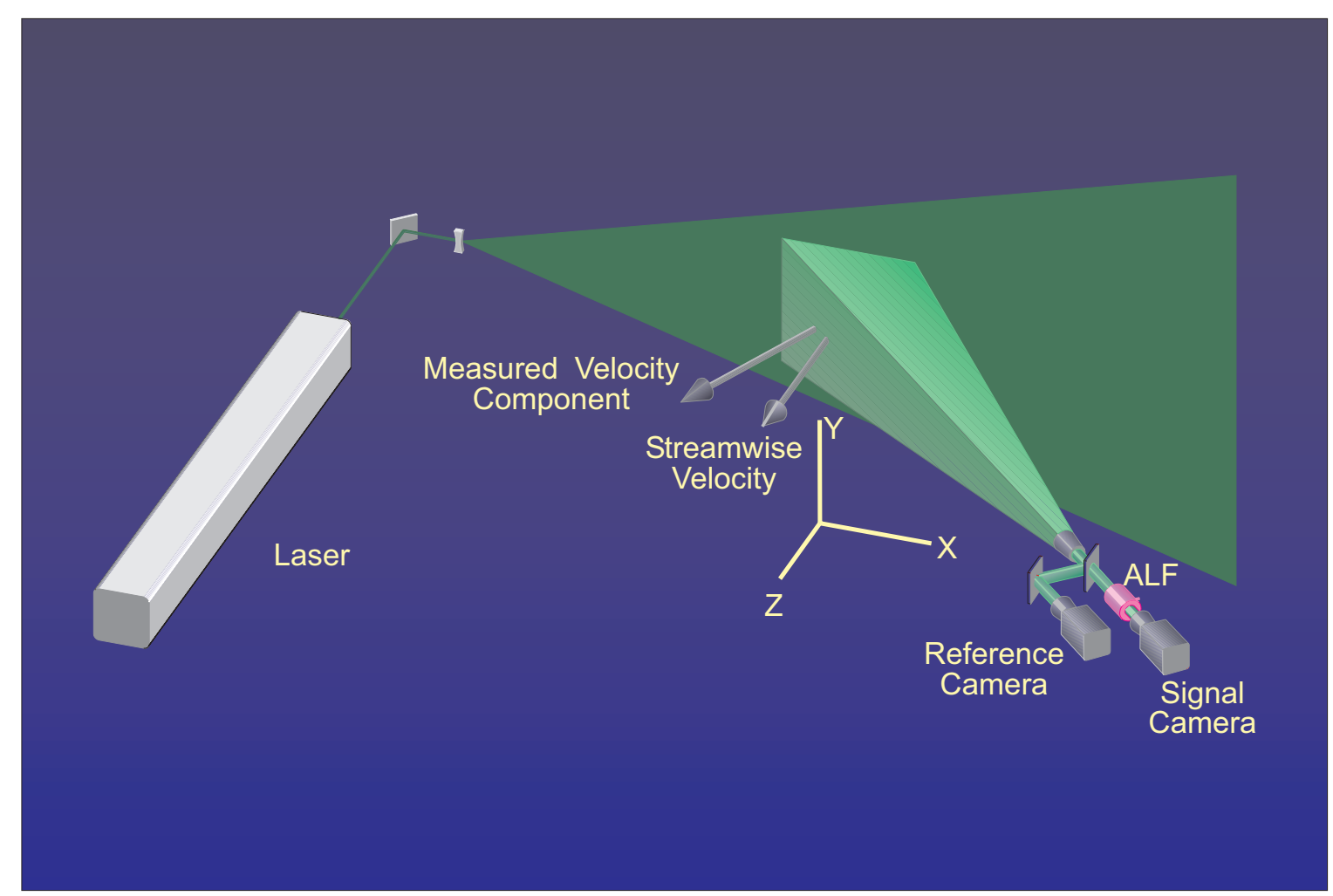

Figure 3.- Pictorial view of the Doppler global velocimeter used in the Basic Aerodynamics Research Tunnel.

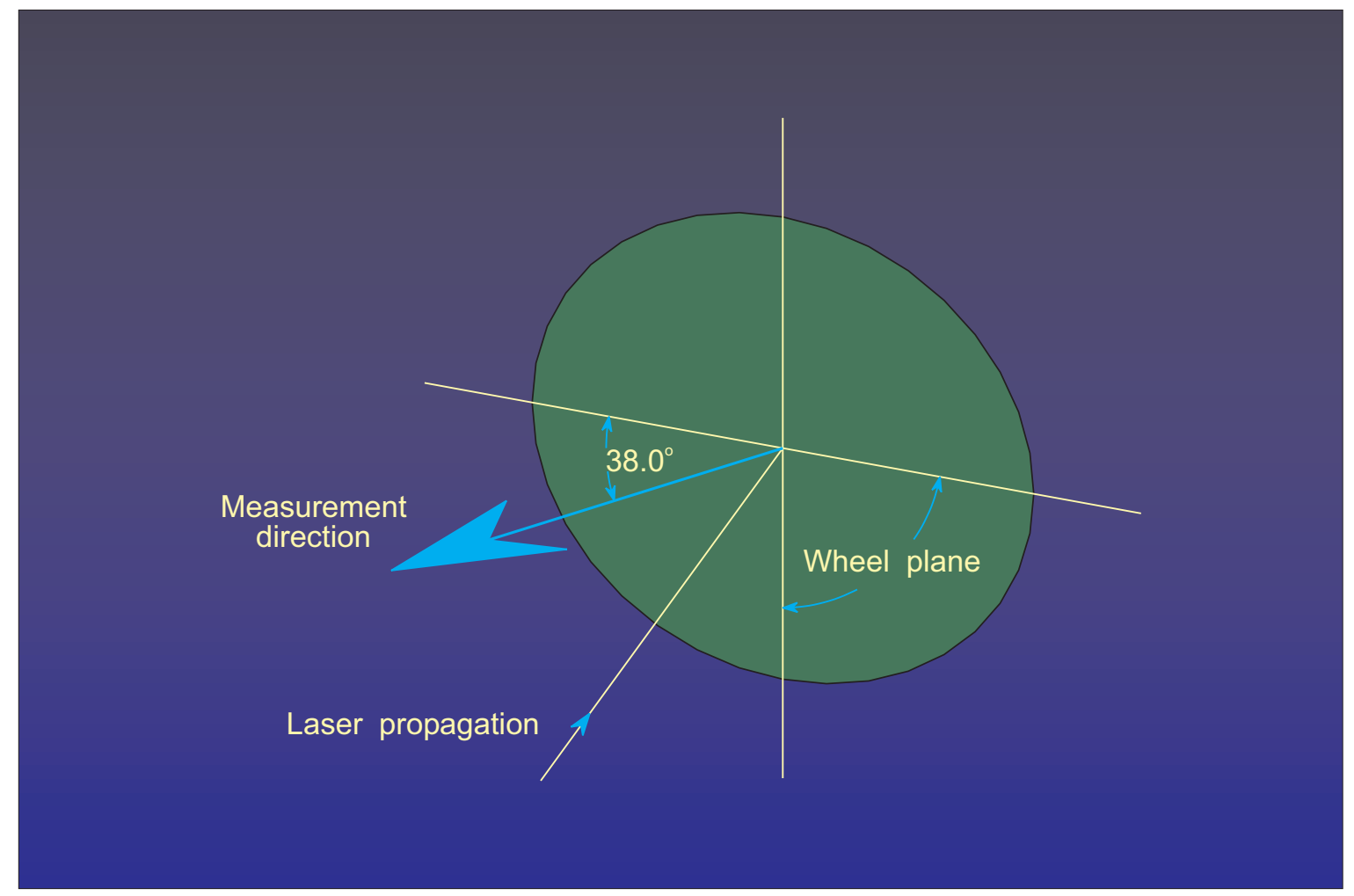

Figure 4.- DGV measurement direction for the wheel experiment. 


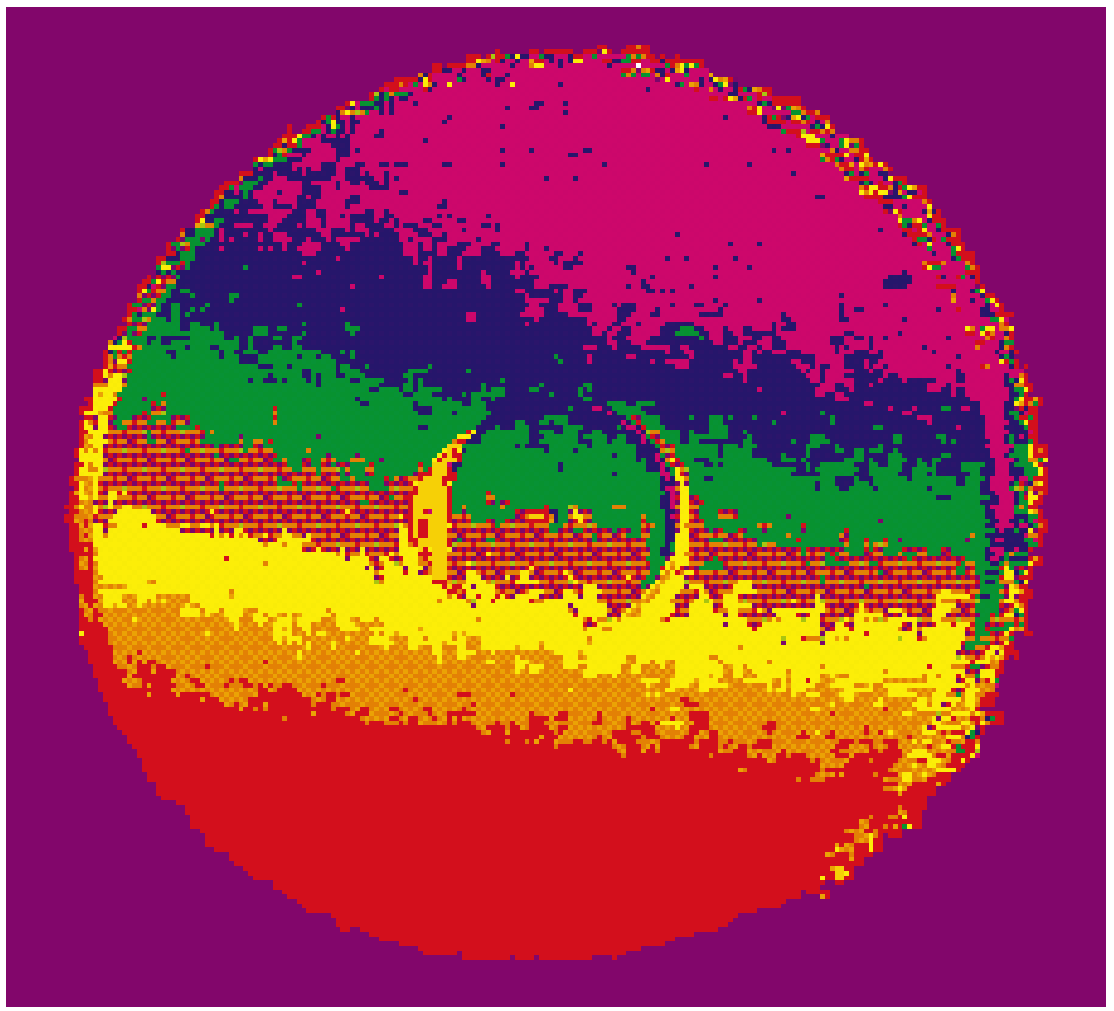

Figure 5.- DGV image map of a rotating wheel.

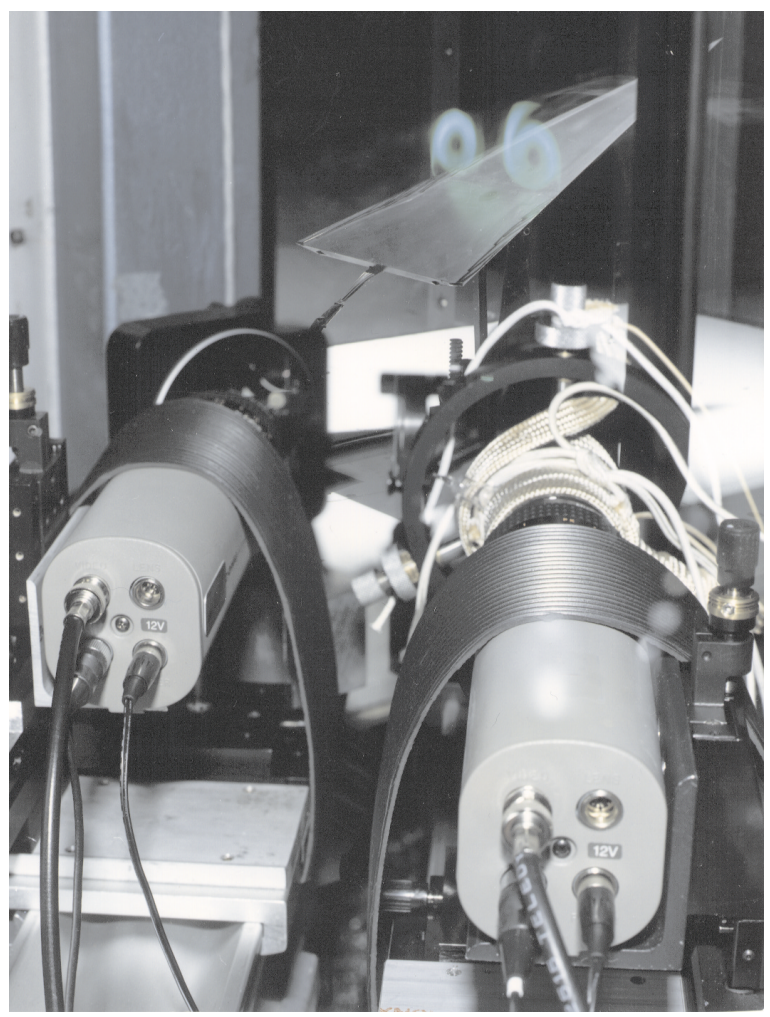

Figure 6.- Photograph of the Doppler global velocimeter installed in the Basic Aerodynamics Research Tunnel. 


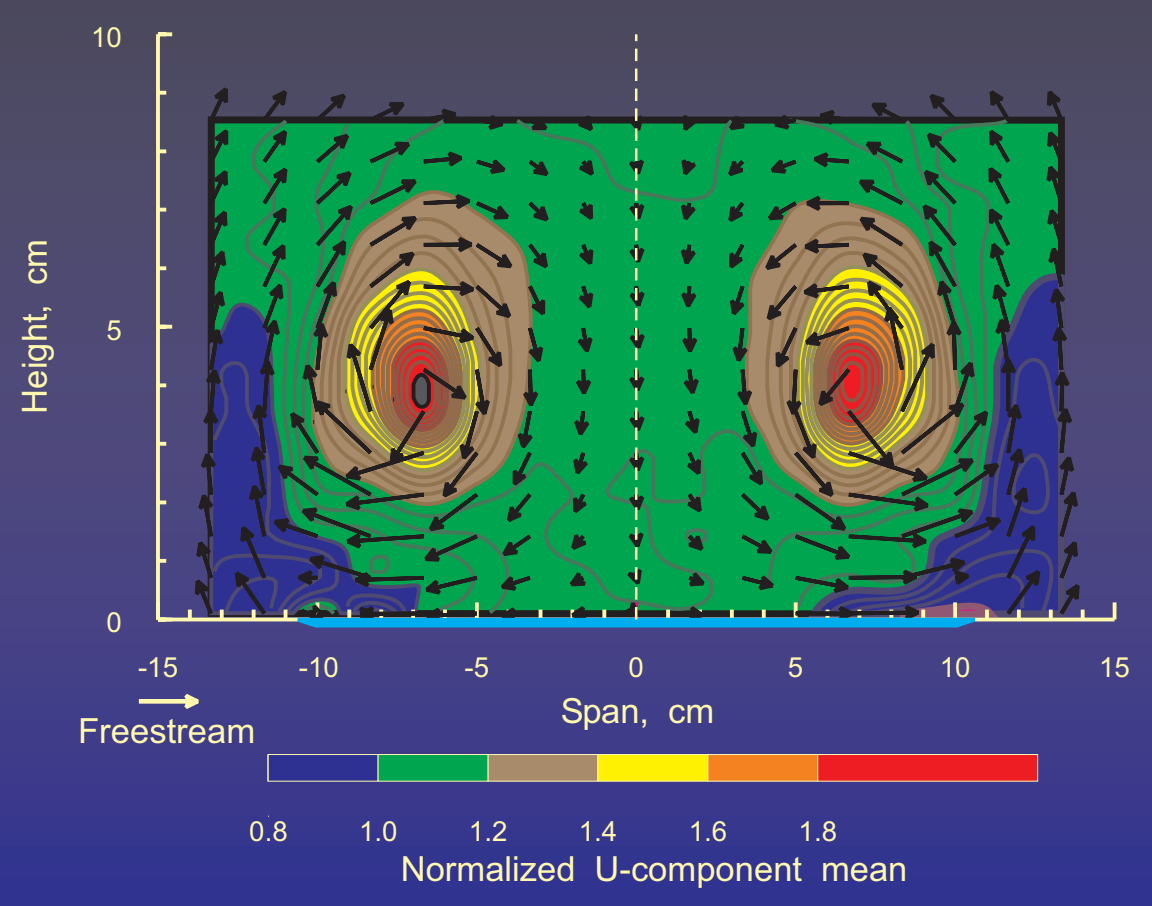

Figure 7.- Three component laser velocimeter measurements of the vortical flow field above a 75-degree delta wing at an angle-of-attack of 20.5 degrees.

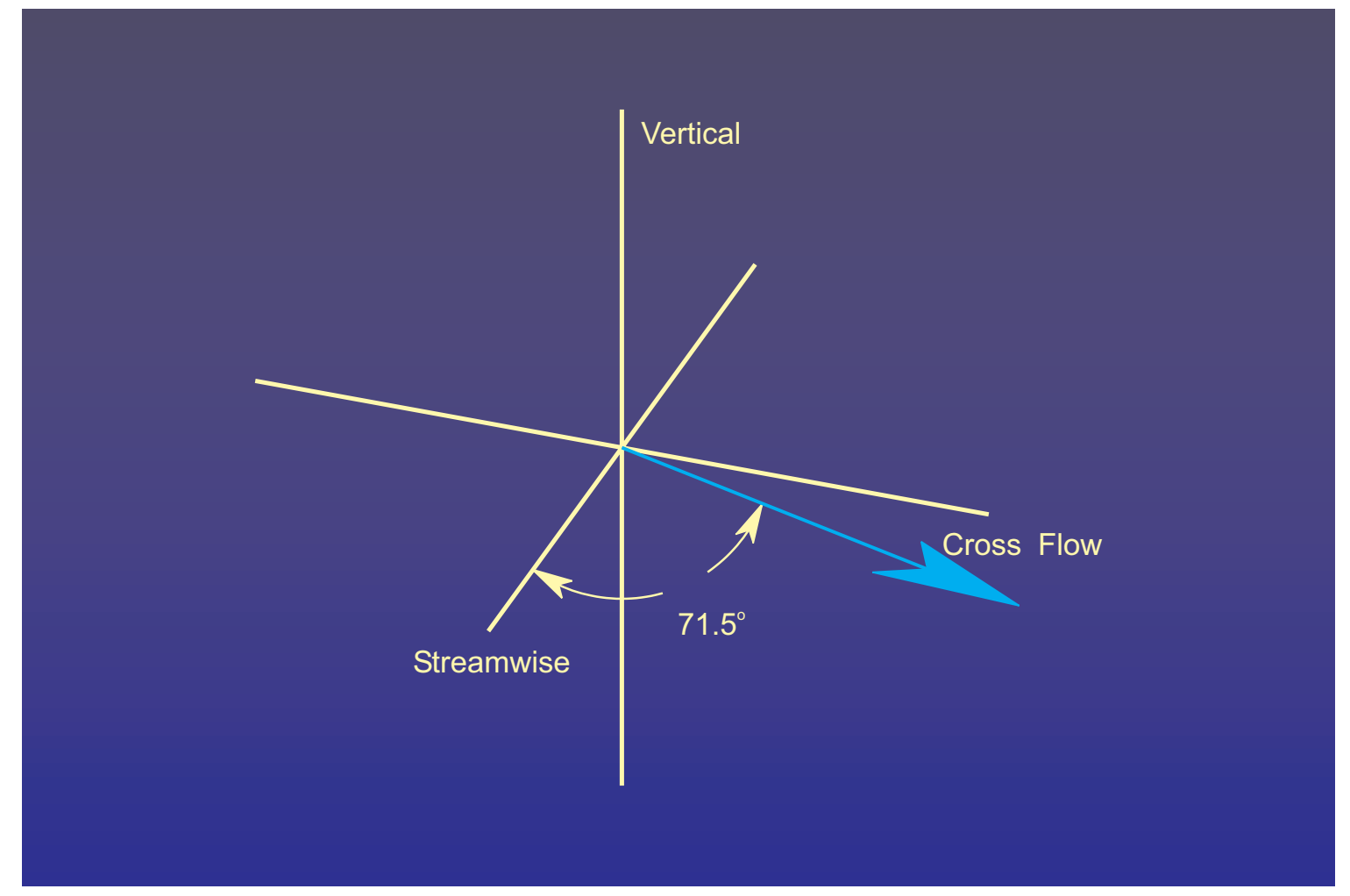

Figure 8.- Measurement direction for DGV operation in backscatter mode. 


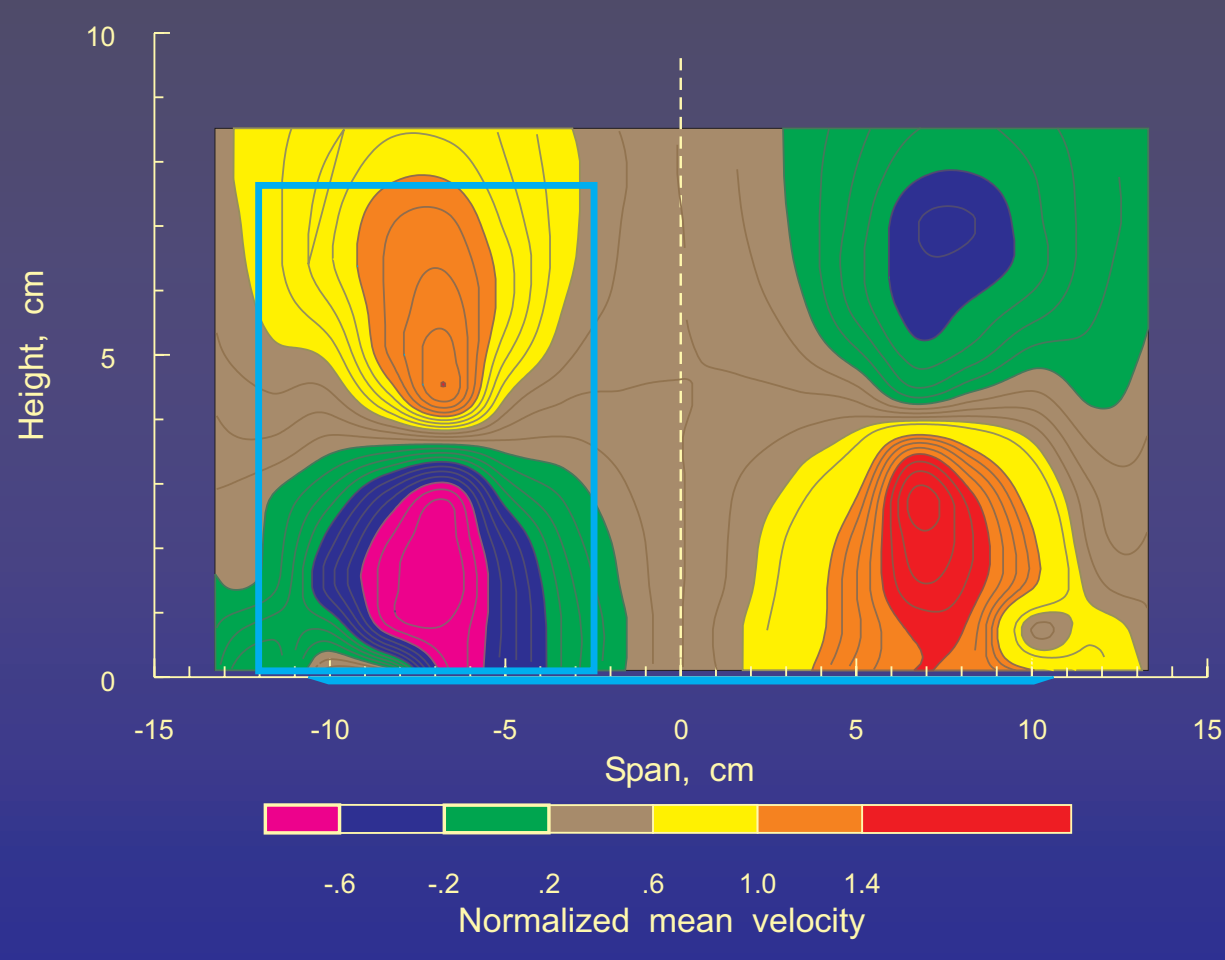

Figure 9.- Resolved laser velocimeter measurements along the direction 71.5 degrees from streamwise in the horizontal plane.

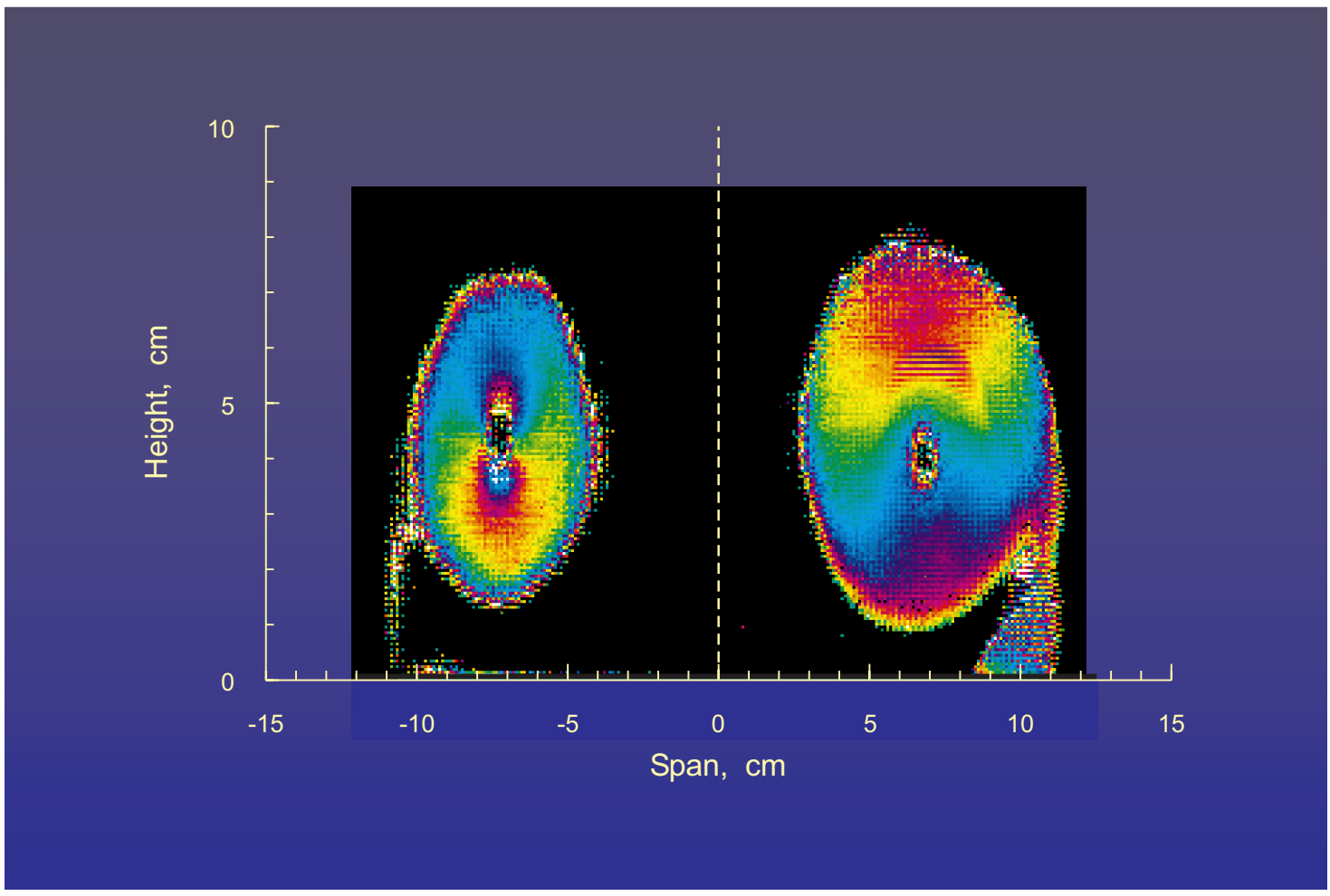

Figure 10.- DGV measurements of the velocity field (average of 30 frames) for the component along the direction 71.5 degrees from streamwise in the horizontal plane. 


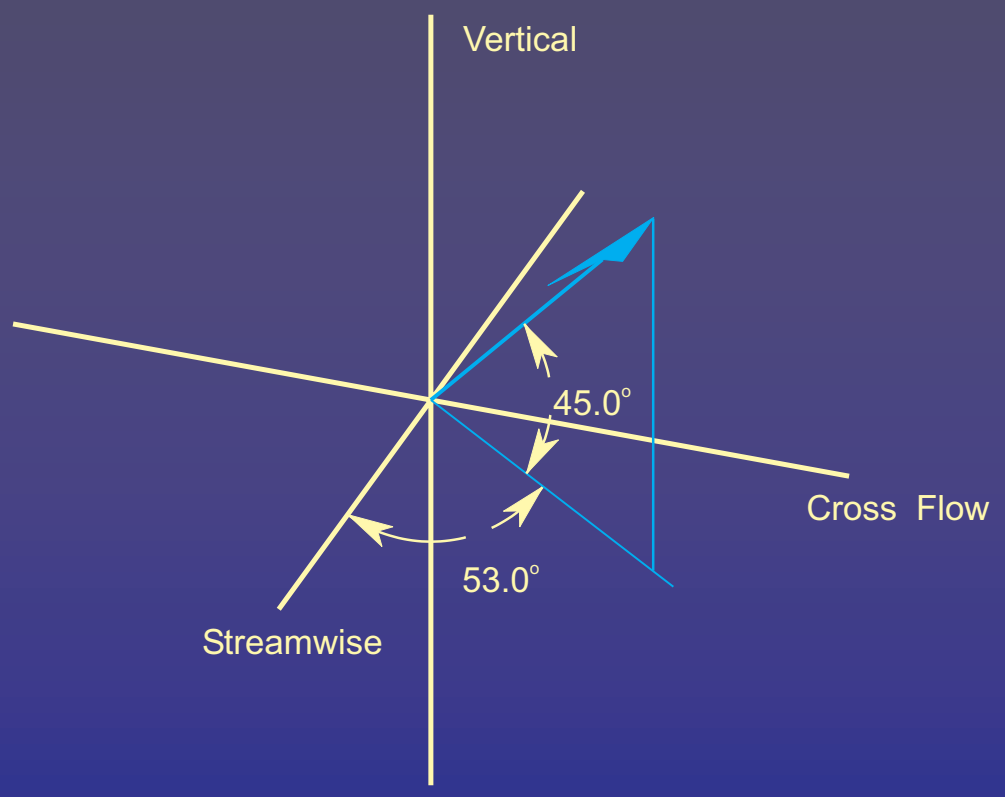

Figure 11.- Measurement direction for DGV operation in side scatter mode, (laser propagation from above).

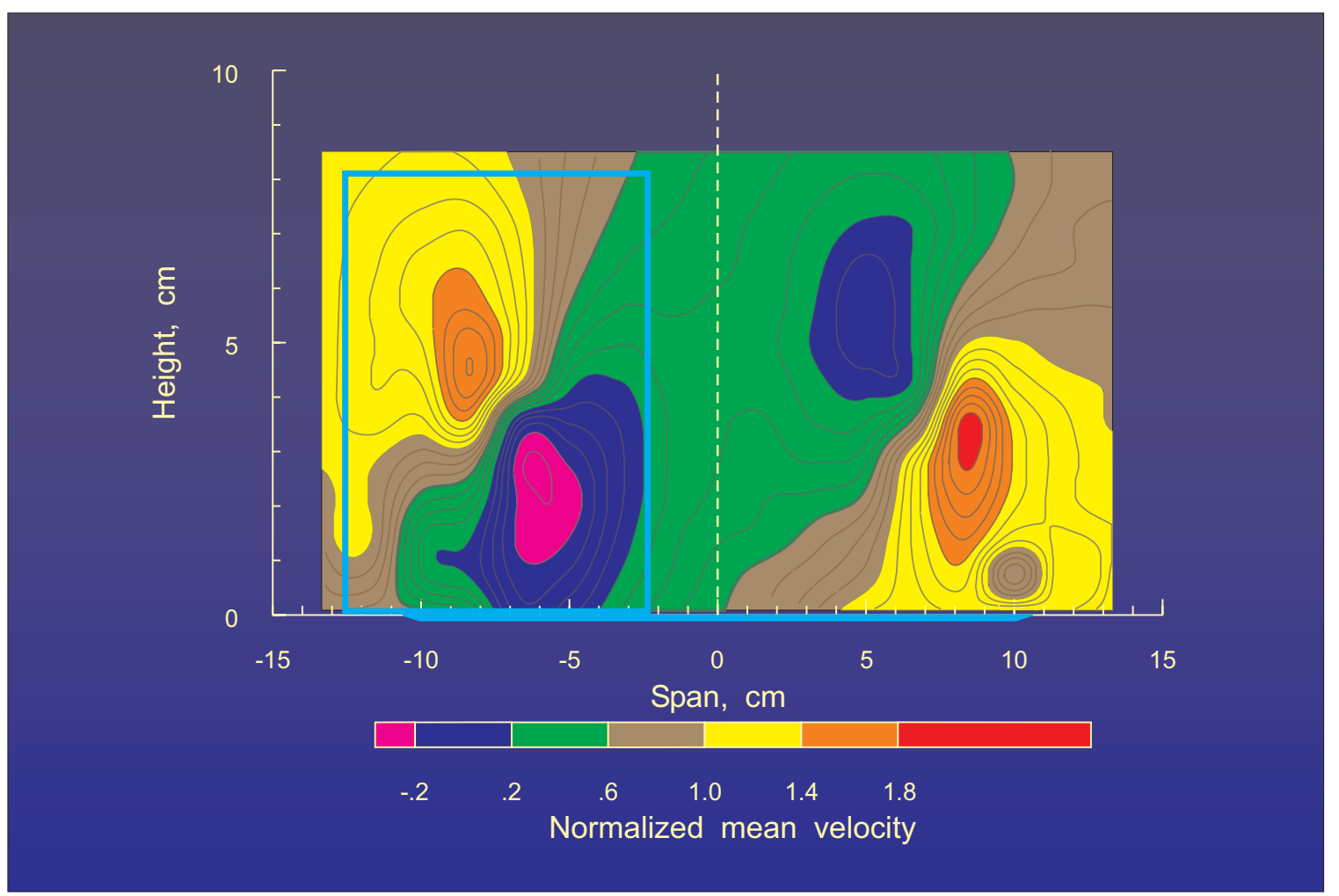

Figure 12.- Resolved laser velocimeter measurements along the direction 53.0 degrees from streamwise and 45.0 degrees above the horizontal plane. 


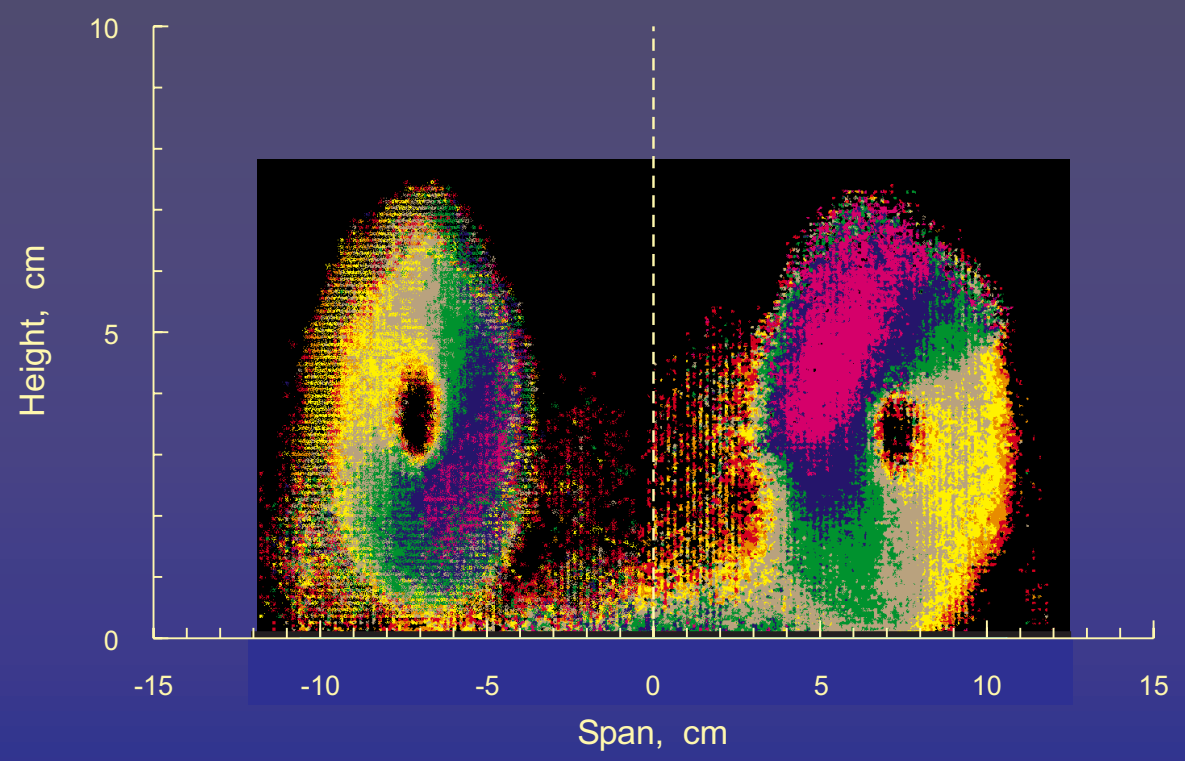

Figure 13.- DGV measurements of the velocity field (average of 30 frames) for the component along the direction 53.0 degrees from streamwise and 45.0 degrees above the horizontal plane.

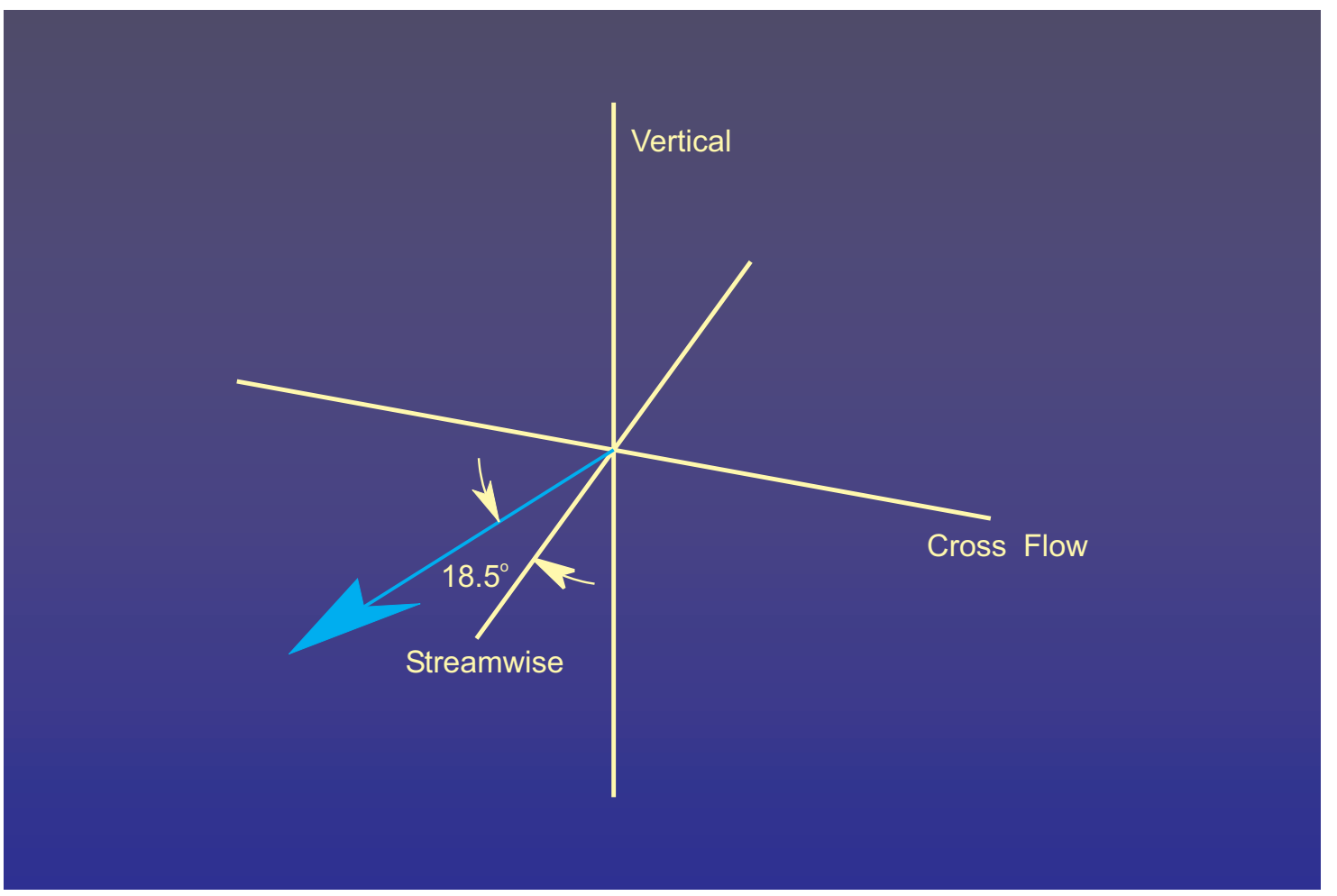

Figure 14.- Measurement direction for DGV operation in forward scatter mode. 


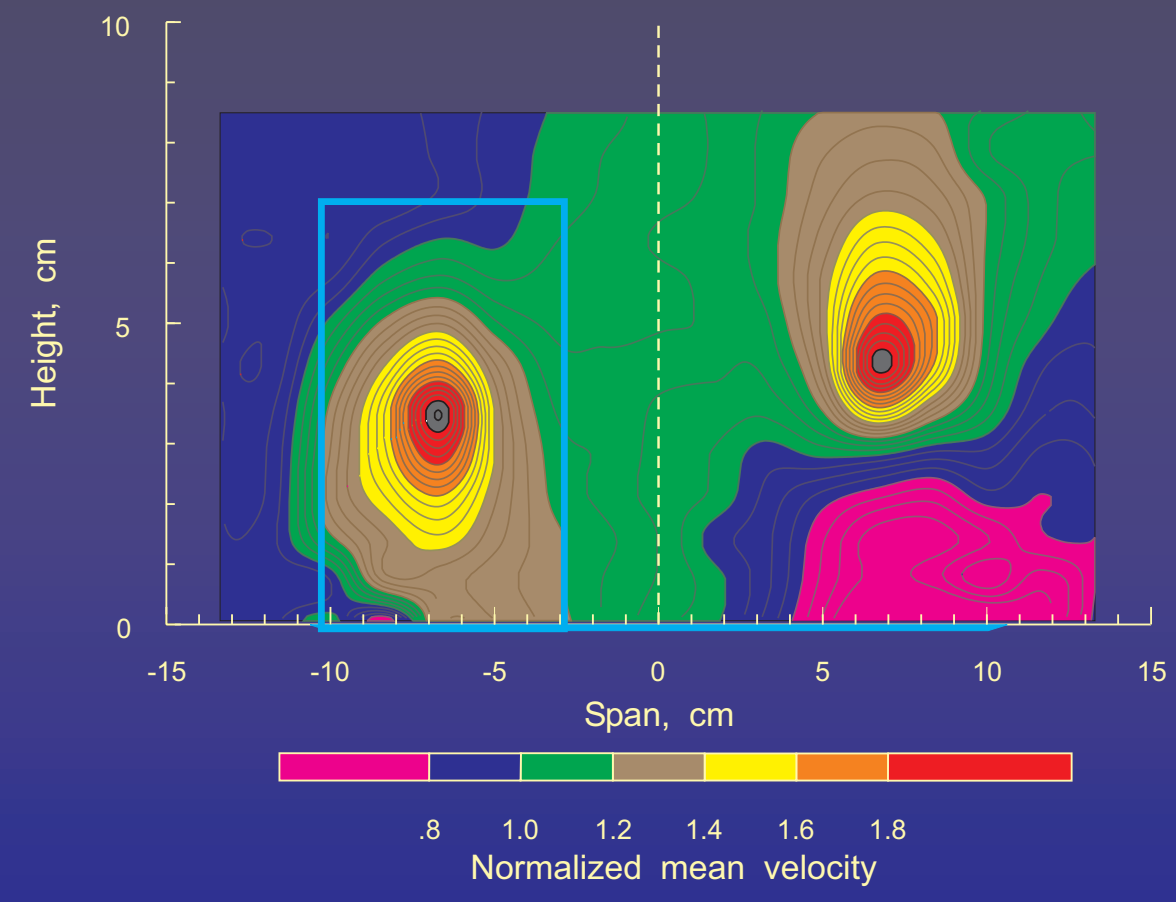

Figure 15.- Resolved laser velocimeter measurements along the direction 18.5 degrees from streamwise in the horizontal plane.

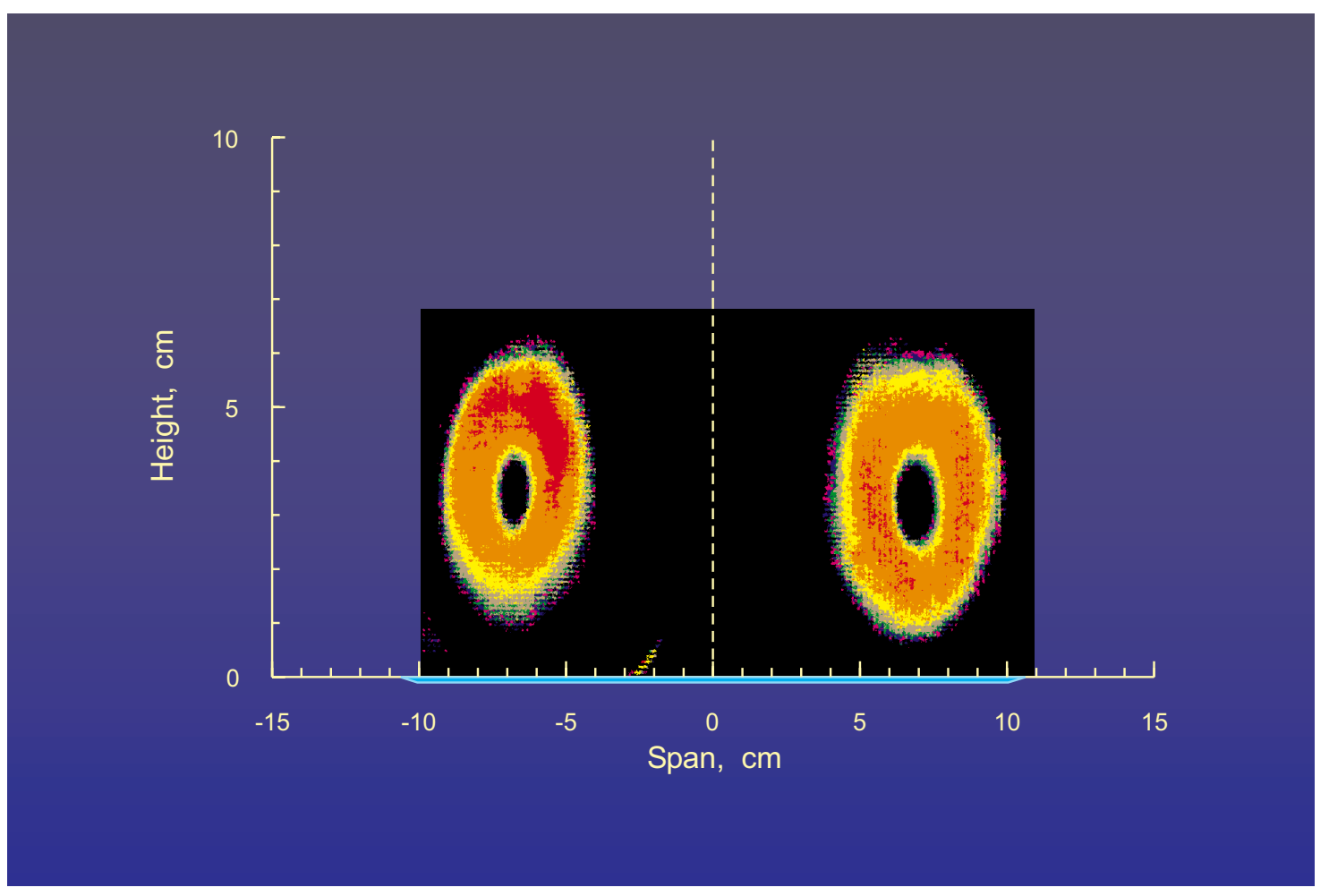

Figure 16.- DGV measurements of the velocity field (average of 30 frames) for the component along the direction 18.5 degrees from streamwise in the horizontal plane. 